\title{
Evaluation of plasma microRNA levels to predict insensitivity of patients with advanced lung adenocarcinomas to pemetrexed and platinum
}

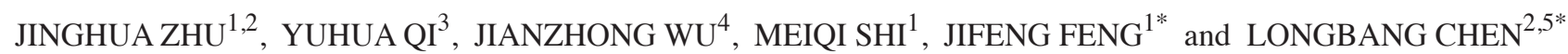 \\ ${ }^{1}$ Department of Medical Oncology, Jiangsu Cancer Institute and Hospital; ${ }^{2}$ School of Medicine, Nanjing University; \\ ${ }^{3}$ Key Laboratories of Enteric Pathogenic Microbiology, Ministry of Health, Microbiological Laboratory, \\ Jiangsu Center for Disease Prevention and Control; ${ }^{4}$ Research Center of Clinical Oncology, \\ Jiangsu Cancer Institute and Hospital; ${ }^{5}$ Department of Medical Oncology, Jinling Hospital, \\ Nanjing, Jiangsu 210002, P.R. China
}

Received July 17, 2015; Accepted September 9, 2016

DOI: $10.3892 / \mathrm{ol} .2016 .5295$

\begin{abstract}
Pemetrexed combined with platinum is a first-line therapy used to treat patients with advanced non-small cell lung cancer (NSCLC) that exhibit negative or unknown epidermal growth factor receptor (EGFR) mutational status or anaplastic lymphoma kinase (ALK) rearrangements. Lung adenocarcinoma (LAC) is the primary type of NSCLC. In order to prevent overtreatment, it is necessary to identify patients with LAC who may not benefit from certain chemotherapies. Patients recruited in the present study $(n=129)$ were diagnosed with advanced LAC and received first-line pemetrexed and platinum-based chemotherapy. A microRNA (miR) microarray was used to screen the plasma miR expression profiles in a screening set of eight patients prior to and following treatment. Specifically, plasma miR-25, miR-21, miR-27b, miR-326, miR-483-5p and miR-920 were selected for reverse transcription-quantitative polymerase chain reaction analysis in a training set $(n=44)$ prior to treatment. The screening and training set patients were all non-smokers with no prior history of serious or chronic disease. The $\Delta \Delta \mathrm{Cq}$ values of these miRs were compared between the group that showed benefit from pemetrexed and platinum treatment and the group that did not. Consequently, the $\Delta \Delta \mathrm{Cq}$ values of miR-25, miR-21, miR-27b and miR-326 were further determined in a validation set $(n=77)$. The results of the present study demonstrate that plasma expression levels of miR-25,
\end{abstract}

Correspondence to: Dr Longbang Chen, Department of Medical Oncology, Jinling Hospital, 315 Zhongshan East Road, Nanjing, Jiangsu 210002, P.R. China

E-mail: chenlongbang@yeah.net

*Contributed equally

Key words: advanced lung adenocarcinoma, pemetrexed, platinum, circulating microRNA, predictive markers
miR-21, miR-27b and miR-326, in the training and validation sets prior to treatment, were significantly different between the benefit and non-benefit groups $(\mathrm{P} \leq 0.001)$. The expression of miR-25, miR-21, miR-27b and miR-326 was upregulated in the non-benefit group and this elevation was positively correlated with decreased progression-free survival (PFS; $\mathrm{P} \leq 0.001)$. In addition, the predictive power of each miR was evaluated through receiver operating characteristic curves, in which miR-25 exhibited the highest degree of accuracy (area under the curve, 0.926 ; 95\% confidence interval, 0.881-0.971). These results indicate that overexpression of plasma miR-25, miR-21, miR-27b and miR-326, prior to treatment, in patients with advanced LAC is predictive of non-benefit from first-line pemetrexed and platinum-based chemotherapy, and is associated with decreased PFS. Among these four miRs, miR-25 exhibited the highest degree of accuracy in predicting insensitivity, suggesting it is the most promising biomarker.

\section{Introduction}

Lung cancer is the primary cause of cancer-associated mortality worldwide (1) and non-small cell lung cancer (NSCLC) accounts for $\sim 85 \%$ of all lung cancer cases. NSCLC is divided into two major types, squamous cell carcinoma and non-squamous cell carcinoma. Non-squamous cell carcinoma is comprised of a number of subtypes, including adenocarcinoma and large-cell carcinoma. Adenocarcinoma is the most frequent non-squamous cell carcinoma and its prevalence is increasing (2).

For patients with advanced NSCLC that exhibit a negative or unknown EGFR mutational status or ALK rearrangements, platinum-based double chemotherapy is the typical first-line therapy $(3,4)$. In the treatment of non-squamous cell carcinoma, pemetrexed combined with platinum demonstrates a significantly improved toxicity profile and OS, compared with taxane- or gemcitabine-based regimes $(3,5,6)$. Nevertheless, a number of patients do not benefit from pemetrexed and platinum-based therapy, due to insensitivity or high toxicity to the combination (7-9). Therefore, it is necessary 
to identify patients unlikely to benefit from this treatment in order to avoid unnecessary treatment. Previous studies have demonstrated that excision repair cross-complementation group 1 (ERCC-1), breast cancer type 1 susceptibility protein (BRCA-1), ribonucleotide reductase catalytic subunit M1 (RRM-1) and thymidylate synthase (TS) are predictive markers of the chemotherapeutic effect of platinum agents, taxanes, gemcitabine and pemetrexed (10-14). However, the predictiveness of ERCC-1, RRM-1 and BRCA-1 has not been reproducible (15-18). There is a particular need for further phase III studies of TS to validate the reproducibility of the applied immunohistochemistry and scoring systems for its use as a predictive marker (18). Consequently, it is important to identify novel biomarkers for the prediction of chemotherapeutic benefit.

MicroRNAs (miRs) are small noncoding RNAs, of between 19 and 25 nucleotides in length, which bind to the 3'-untranslated region of mRNAs to regulate their translation. miRs are involved in numerous essential biological processes and are associated with a number of types of cancer and disease (19). Numerous studies have demonstrated that miRs are dysregulated in cancer, such as that of the lung (20-23). As circulating miRs are stable in blood plasma, they have potential applications as diagnostic markers $(24,25)$. Increasing evidence has shown circulating miR profile analysis can aid in early diagnosis, staging, tracking and prognosis of different types of cancer (26-32). In addition, circulating miR profile analysis is useful in predicting the response to chemotherapy $(33,34)$.

The present study aimed to determine which plasma miRs may be used to predict the clinical outcome of pemetrexed and platinum treatment for advanced lung adenocarcinoma (LAC). In contrast with previous research, patient benefit to the treatment was examined instead of tumor response. Seidman et al (35) observed that the overall survival (OS) of patients with stable metastatic breast cancer resembled that of patients with complete remission (CR) or partial remission (PR), indicating that similar benefits to remission may come from stable disease (SD). Therefore, benefit $(\mathrm{CR}+\mathrm{PR}+\mathrm{SD})$ may be deemed a more accurate indicator of treatment efficacy than tumor response (36). A miR microarray and reverse transcription-quantitative polymerase chain reaction (RT-qPCR) were used to identify and verify potential markers in training and validation sets following screening. It was demonstrated that high plasma expression levels of miR-25, miR-21, miR-27b and miR-326 may predict non-benefit from chemotherapy, and that increased levels of these miRs was inversely correlated with progression-free survival (PFS).

\section{Materials and methods}

Study participants. A total of 129 participants (Table I) diagnosed with stage IIIB-IV LAC were recruited from the Jiangsu Cancer Institute and Hospital (Nanjing, China) between September 2010 and January 2013. All patients had histological or cytological confirmation of their tumor diagnosis. Tumors were staged based on the Seventh Edition Tumor-Node-Metastasis Staging System of the American Joint Committee on Cancer (37). All patients received first-line chemotherapy, pemetrexed $\left(500 \mathrm{mg} / \mathrm{m}^{2}\right)$ on day 1 with either cisplatin $\left(75 \mathrm{mg} / \mathrm{m}^{2}\right)$ or carboplatin [area under the curve
$(\mathrm{AUC})=5$ ] on day 2 of a 21-day treatment cycle. All patients experienced $\geq 2$ cycles of chemotherapy. Therapeutic response was evaluated by computed tomography following 2 cycles of treatment, according to Response Evaluation Criteria in Solid Tumors 1.1 (38). Response was classified as PR, CR, SD or progressive disease (PD). Patients classified as CR, PR or SD for $\geq 4$ weeks were assigned to the benefit group. Conversely, patients classified as PD were assigned to the non-benefit group.

Plasma samples from all patients were collected prior to chemotherapy, between September 2010 and January 2013. A miR microarray was used to screen the plasma miR expression profiles of a screening set of eight patients prior to and following treatment. Specifically, plasma miR-25, miR-21, miR-27b, miR-326, miR-483-5p and miR-920 were selected for analysis in a training set $(n=44)$ prior to treatment. The screening and training set patients were all non-smokers with no prior history of serious or chronic disease. Consequently, the $\Delta \Delta \mathrm{Cq}$ values of miR-25, miR-21, miR-27b and miR-326 were further determined in a validation set $(n=77)$, which included smokers and patients with a prior history of serious or chronic disease. Patients were observed until December 2014. The present study was approved by the Medical Ethics Committee of Jiangsu Cancer Institute and Hospital and all participants provided informed consent.

Total RNA isolation. Whole blood samples ( $5 \mathrm{ml}$ per patient) were collected in anticoagulant tubes and centrifuged at $1,811 \times \mathrm{g}$ for $5 \mathrm{~min}$ at $4^{\circ} \mathrm{C}$. The supernatant was subsequently transferred into an RNase/DNase-free $1 \mathrm{ml}$ microfuge tube and immediately stored at $-80^{\circ} \mathrm{C}$ until required. As there is no established endogenous miR control for blood plasma, prior to the isolation process, cel-miR-238 (UUUGUACUCCGA UGCCAUUCAGA; Takara Biotechnology Co., Ltd., Dalian, China), a synthetic Caenorhabditis elegans miR, was mixed with every sample as an internal control, at a final concentration of 25 fmol. Separation of total RNA from the plasma was performed using the NucleoSpin miRNA Plasma kit (Machery-Nagel GmbH, Düren, Germany), according to the manufacturer's instructions. RNA purity and quantity was determined using a NanoDrop spectrophotometer (ND-1000; Thermo Fisher Scientific, Inc., Waltham, MA, USA).

miR microarray analysis. The isolated RNAs were labeled using the miRCURY Hy3/Hy5 Power Labeling kit (cat. no. 208520), and the Hy3TM-labeled samples were then hybridized, following the manufacturer's protocol, to the miRCURYTM LNA Array (cat. no. 208031-A) (both Exiqon A/S, Vedbaek, Denmark). This array covered miRs annotated in miRBase version 18.0 (www.mirbase.org), comprising of 3,100 capture probes, including all human, rat and mouse miR genes. Subsequently, the Axon GenePix 4000B Microarray Scanner (Molecular Devices, LLC, Sunnyvale, CA, USA) was used to scan the slides at a wavelength of $532 \mathrm{~nm}$. Image files were then transferred to GenePix Pro version 6.0 software (Molecular Devices, LLC) for further analysis. Replicated miR readings were averaged and miRs with intensities $\geq 50$ were selected for normalization using the median normalization method (39). Subsequently, fold change filtering identified differentially expressed miRs. 
Table I. Demographic and clinical characteristics of the non-benefit and benefit groups.

\begin{tabular}{|c|c|c|c|c|c|c|c|c|}
\hline \multirow[b]{2}{*}{ Characteristic } & \multicolumn{2}{|c|}{ Profiling set } & \multicolumn{3}{|c|}{ Testing set } & \multicolumn{3}{|c|}{ Validation set } \\
\hline & Non-benefit & Benefit & Non-benefit & Benefit & P-value & Non-benefit & Benefit & P-value \\
\hline Number of patients & 4 & 4 & 15 & 29 & & 26 & 51 & \\
\hline Mean age, (years \pm standard error) & $43 \pm 1.2$ & $45 \pm 4.4$ & $55 \pm 10.1$ & $56 \pm 8.8$ & $0.717^{\mathrm{a}}$ & $60 \pm 10.1$ & $55 \pm 11.2$ & $0.159^{\mathrm{a}}$ \\
\hline \multicolumn{9}{|l|}{ Gender (no.) } \\
\hline Male & 3 & 3 & 10 & 18 & $0.764^{\mathrm{b}}$ & 18 & 31 & $0.466^{\mathrm{b}}$ \\
\hline Female & 1 & 1 & 5 & 11 & & 8 & 20 & \\
\hline \multicolumn{9}{|l|}{ Stage of LAC } \\
\hline IIIB & 0 & 0 & 2 & 3 & $1.000^{\mathrm{c}}$ & 3 & 7 & $0.617^{\mathrm{c}}$ \\
\hline IV & 4 & 4 & 13 & 26 & & 23 & 44 & \\
\hline \multicolumn{9}{|l|}{ Smoking status } \\
\hline Yes & 0 & 0 & 0 & 0 & & 10 & 21 & $0.818^{\mathrm{b}}$ \\
\hline No & 4 & 4 & 15 & 29 & & 16 & 30 & \\
\hline \multicolumn{9}{|l|}{ EGFR genotype } \\
\hline Wild-type & 1 & 0 & 1 & 3 & $1.000^{\mathrm{c}}$ & 4 & 5 & $0.477^{\mathrm{c}}$ \\
\hline Unknown & 3 & 4 & 14 & 26 & & 22 & 46 & \\
\hline \multicolumn{9}{|c|}{ Prior serious or chronic disease history } \\
\hline Yes & 0 & 0 & 0 & 0 & & 11 & 22 & $0.945^{\mathrm{b}}$ \\
\hline No & 4 & 4 & 15 & 29 & & 15 & 29 & \\
\hline \multicolumn{9}{|l|}{ KPS score } \\
\hline$\geq 80$ & 4 & 4 & 15 & 29 & & 26 & 51 & \\
\hline$<80$ & 0 & 0 & 0 & 0 & & 0 & 0 & \\
\hline \multicolumn{9}{|l|}{ Relative dose intensity (\%) } \\
\hline Pemetrexed & 100.0 & 97.0 & 98.0 & 97.7 & & 98.5 & 97.6 & \\
\hline Carboplatin & 71.5 & 62.5 & 91.0 & 78.7 & & 87.2 & 86.1 & \\
\hline Cisplatin & 87.0 & 100.0 & 93.2 & 92.6 & & 94.1 & 91.6 & \\
\hline \multicolumn{9}{|l|}{ Maintenance therapy } \\
\hline Yes & 0 & 2 & 0 & 19 & & 0 & 24 & \\
\hline No & 4 & 2 & 15 & 10 & & 26 & 27 & \\
\hline
\end{tabular}

${ }^{a}$ Two-sided $\chi^{2}$ test; ${ }^{\text {PPearson's }} \chi^{2}$ test; ${ }^{\mathrm{c}}$ Fisher's exact test. LAC, lung adenocarcinoma; EGFR, epidermal growth factor receptor; KPS, Karnofsky performance status.

All miR microarray analysis was performed by KangChen Bio-tech (Shanghai, China).

Quantification and confirmation of candidate miRs using $R T$-qPCR. Plasma sample miRs were quantified using RT-qPCR. Briefly, $1.67 \mu \mathrm{l}$ RNA (50 ng) was included in a $5 \mu \mathrm{l}$ reaction mixture using the TaqMan MicroRNA Reverse Transcription kit (Applied Biosystems; Thermo Fisher Scientific, Inc.) and a RT primer (TaqMan ${ }^{\circledR}$ MicroRNA Assay; Applied Biosystems; Thermo Fisher Scientific, Inc.) to convert miRNA to cDNA. The RT thermocycling conditions were as follows: $30 \mathrm{~min}$ at $16^{\circ} \mathrm{C} ; 30 \mathrm{~min}$ at $42^{\circ} \mathrm{C} ; 5 \mathrm{~min}$ at $85^{\circ} \mathrm{C}$; and $4^{\circ} \mathrm{C}$ until required. Subsequently, qPCR was carried out in a volume of $10 \mu \mathrm{l}$ containing $4.5 \mu \mathrm{l}$ diluted cDNA (1:15), $5 \mu \mathrm{l}$ TaqMan Universal PCR Master Mix (No AmpErase UNG), AmpliTaq Gold $^{\circledR}$ DNA Polymerase and $0.5 \mu$ l PCR probes (TaqMan ${ }^{\circledR}$ MicroRNA Assay; Applied Biosystems; Thermo Fisher Scientific, Inc.). qPCR was performed using a 7900 Fast Real-Time
PCR system (Applied Biosystems; Thermo Fisher Scientific, Inc.). qPCR thermocycling conditions were as follows: $10 \mathrm{~min}$ at $95^{\circ} \mathrm{C} ; 40$ cycles of $95^{\circ} \mathrm{C}$ for $15 \mathrm{sec}$; and $60^{\circ} \mathrm{C}$ for $1 \mathrm{~min}$. Each sample was run in duplicate and the mean $\mathrm{Cq}$ value was determined. The relative miRs expression level $\left(\log _{2}\right.$ relative expression level) was calculated using the $2^{-\Delta \Delta \mathrm{Cq}}$ method (40), where $\Delta \mathrm{Cq}$ was calculated as follows: $\mathrm{Cq}$ (target $\mathrm{miR})-\mathrm{Cq}$ (cel-miR-238).

Statistical analysis. Fisher's exact test, a two-sided $\chi^{2}$ test and Pearson's $\chi^{2}$ test were used to evaluate differences in the clinicopathological factors between groups. To compare the expression levels of different miRs between the benefit and non-benefit groups, the Mann-Whitney U test was used. To estimate the diagnostic potential of plasma miRs, receiver-operating characteristics (ROC) curves were produced. The predictive power was estimated by calculating the AUC of the ROCs, and the maximum value of the Youden's index was used as a criterion 
Table II. Relative plasma expression of miRNAs from microarray analysis.

Relative plasma expression (fold change)

\begin{tabular}{lccr}
\cline { 2 - 3 } miR type & $\begin{array}{c}\text { B prior to treatment: } \\
\text { NB prior to treatment }\end{array}$ & $\begin{array}{c}\text { B prior to treatment: } \\
\text { B following treatment }\end{array}$ & $\begin{array}{r}\text { NB prior to treament: } \\
\text { NB following treatment }\end{array}$ \\
\hline miR-21 & 0.018830 & 16.524080 & 0.474396 \\
miR-27b & 0.013600 & 40.662910 & $<2.000000$ \\
miR-326 & 0.030405 & $<2.000000$ & 0.483120 \\
miR-25 & 0.093175 & 12.106610 & $<2.000000$ \\
miR-483-5p & 17.768710 & 0.109332 & 4.362240 \\
miR-920 & 22.678370 & 0.027606 & 2.048308
\end{tabular}

miR, microRNA; B, benefit; NB, non-benefit.

for selecting the optimum cut-off point (41). The Kaplan-Meier estimator was used to evaluate PFS and compare log rank statistics. SPSS software (version 19.0; IBM SPSS, Armonk, NY, USA) was used for statistical analysis. Results are presented as the mean \pm standard deviation. $\mathrm{P}<0.05$ was considered to indicate a statistically significant difference.

\section{Results}

Patient characteristics. Patient characteristics are presented in Table I. The demographics and clinical characteristics of patients in the benefit and non-benefit groups were similar in regards to age, gender, smoking status, tumor stage, EGFR/ALK state, prior serious/chronic disease history and Karnofsky performance status score. The relative dose intensity of each drug was the real dose (actual dose used): Ideal dose (the dose planned) ratio. PFS time was calculated from the date of initiation of chemotherapy to the date of the last follow-up, to the date of detected progression or date of death owing to any cause. Among the patients, 13 could not be evaluated for PFS due to requiring further radiotherapy or being untraceable for follow-ups.

miR expression profiles in the plasma of patients with LAC prior to and following chemotherapy. miR expression profiles were assessed in 8 patients (benefit group, $n=4$; non-benefit group, $\mathrm{n}=4$ ), prior to and following treatment, in duplicate, to give a total of 16 plasma samples. The results showed that, prior to treatment, there were 312 upregulated miRs (fold change $\geq 2.0$ ) in the benefit group and 233 upregulated miRs in the non-benefit group. Following treatment, there were 213 upregulated and 186 downregulated miRs in the benefit group, and 188 upregulated and 157 downregulated miRs in the non-benefit group. The miRs selected for further confirmation by RT-qPCR displayed the following: A 10-fold change in expression between the benefit and non-benefit groups; $>2$-fold change in expression in the same group between pre- and post-treatment; and an association with cancer in published literature. A total of 6 of the differentially expressed miRs (miR-483-5p, miR-920, upregulated in the benefit group; miR-21, miR-27b, miR-326, miR-25, upregulated in the non-benefit group) were further analyzed. Relative expressions of these 6 miRs are listed in Table II. $m i R$ expression in the training set. RT-qPCR was used to confirm the expression levels of 6 candidate miRs in a training set (benefit, $n=29$; non-benefit, $n=15$ ). To avoid the effect of compounding factors, all patients recruited were non-smokers with no prior history of serious or chronic disease. The $\Delta \Delta \mathrm{Cq}$ values and relative expression levels of the candidate miRs was calculated (Fig. 1A), confirming the screening results of miR-25, miR-27b, miR-21 and miR-326. However, the relative expression levels of miR-483-5p and miR-920 were inconsistent with the results of the microarray (data not shown). As shown in Fig. $1 \mathrm{~A}$, the $\Delta \Delta \mathrm{Cq}$ values of miR-25, miR-27b, miR-21 and miR-326 in patient plasma prior to treatment were significantly increased in the benefit group compared with the non-benefit group $(\mathrm{P}<0.001)$. Therefore, miR-25, miR-27b, miR-21 and miR-326 were selected for further validation.

Confirmation of miR expression in the validation set. As shown in Fig. $1 \mathrm{~B}$, the $\Delta \Delta \mathrm{Cq}$ values of plasma miR-25, miR-27b, miR-21 and miR-326 prior to treatment were compared between the benefit and non-benefit groups in a validation set (benefit, $n=51$; non-benefit, $n=26$ ). The $\Delta \Delta \mathrm{Cq}$ values of these $4 \mathrm{miRs}$ were significantly upregulated in the benefit group compared with the non-benefit group $(\mathrm{P} \leq 0.001$; Fig. 1B). In addition, the trend in the alteration of relative expression levels was similar to that seen in the training set.

Evaluation of the diagnostic potential of the candidate miRs. ROC curve analysis was performed on miR-25, miR-27b, miR-21 and miR-326 in 121 patients to evaluate their suitability as predictive biomarkers of pemetrexed and platinum insensitivity (Fig. 2). The AUC of miR-25 was 0.926 [95\% confidence interval (CI), 0.881-0.971], which was the highest of the four miRs tested. The AUCs of miR-21, miR-27b and miR-326 were 0.905 (95\% CI, 0.845-0.964), 0.817 (95\% CI, 0.733-0.900) and 0.803 (95\% CI, 0.717-0.890), respectively. The optimal cut-off points were-0.171 (sensitivity, 91.3\%; specificity, $80.5 \%$ ) for miR-25, 0.568 (sensitivity, 82.5\%; specificity, $73.2 \%$ ) for miR-27b, -1.85 (sensitivity, $82.5 \%$; specificity, $85.4 \%$ ) for mir-21 and 3.05 (sensitivity, $90.0 \%$; specificity, $63.4 \%$ ) for mir-326. miR-25 exhibited the most accurate predictive power. 
A

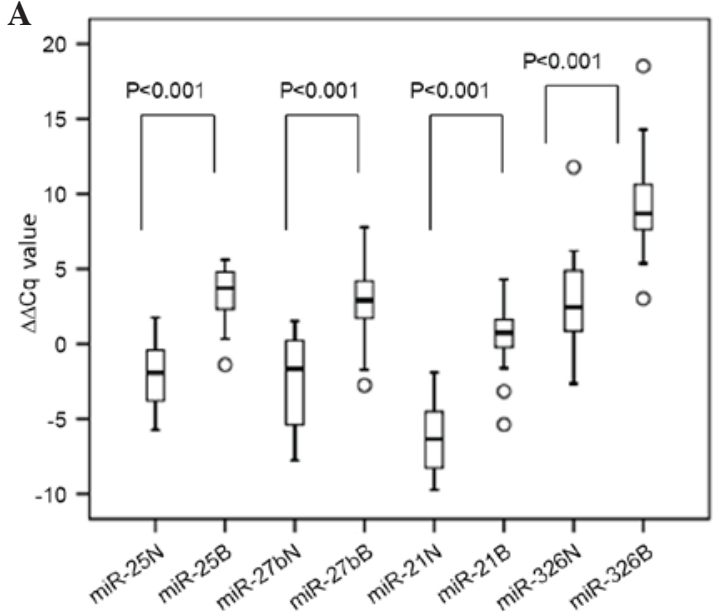

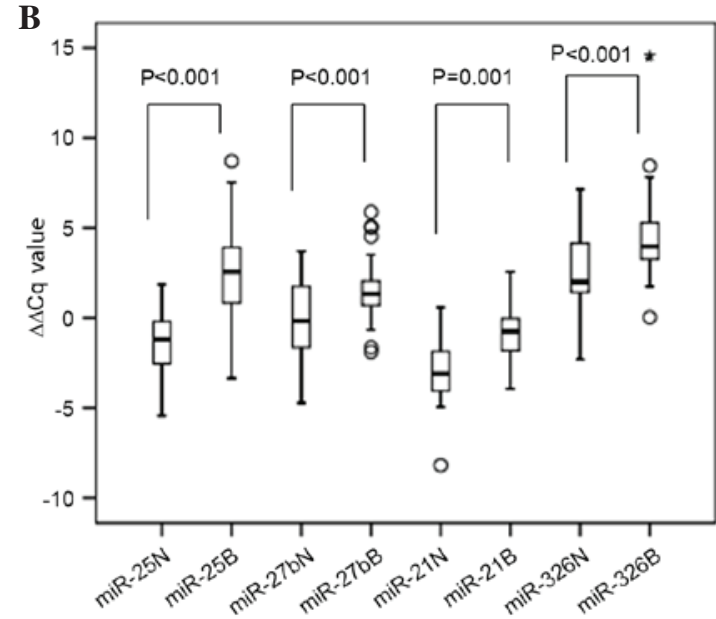

Figure 1. Box-plots of plasma miR-25, miR-27b, miR-21 and miR-326 expression $\Delta \Delta$ Cq values of the non-benefit and benefit groups in the (A) training set $(\mathrm{n}=44)$, and $(\mathrm{B})$ validation set $(\mathrm{n}=77)$. Plasma miR expression levels were analyzed by reverse transcription-quantitative polymerase chain reaction analysis and $\Delta \Delta \mathrm{Cq}$ values were compared using the Mann-Whitney $\mathrm{U}$ test. $\Delta \Delta \mathrm{Cq}$ values for the 4 plasma miRs, prior to treatment, were significantly upregulated in the benefit group compared with the non-benefit group, in the training set and validation sets (o, outlier; ${ }^{*}$, abnormal value). Outliers: the deviation between the measured value and the average value is less than two times the standard deviation; abnormal: the deviation between the measured value and the average value is more than two times the standard deviation. miR, microRNA; N, non-benefit group; B, benefit group.

A

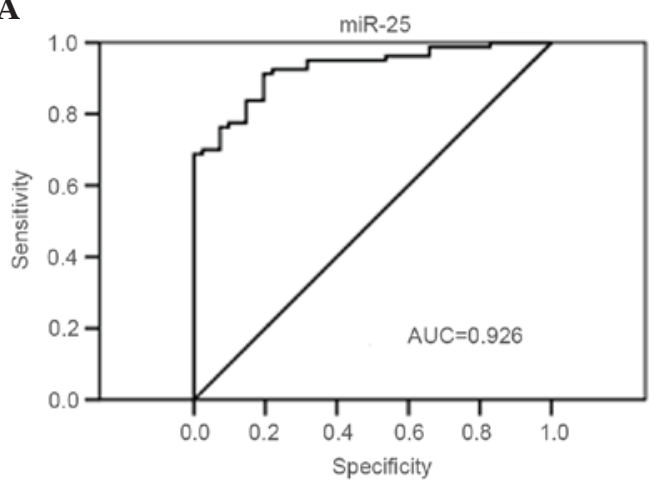

C

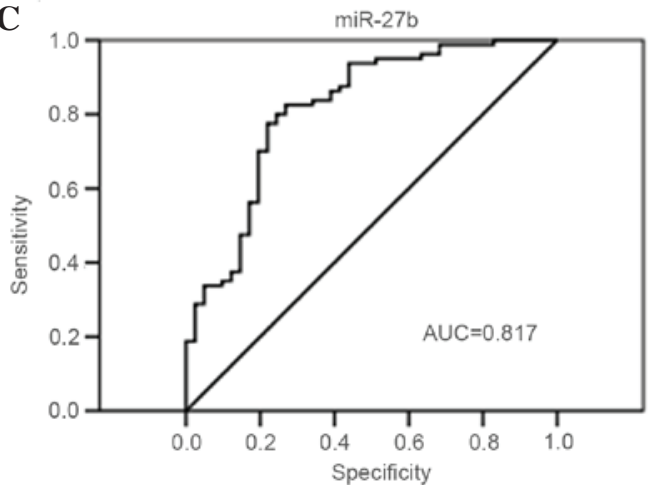

B

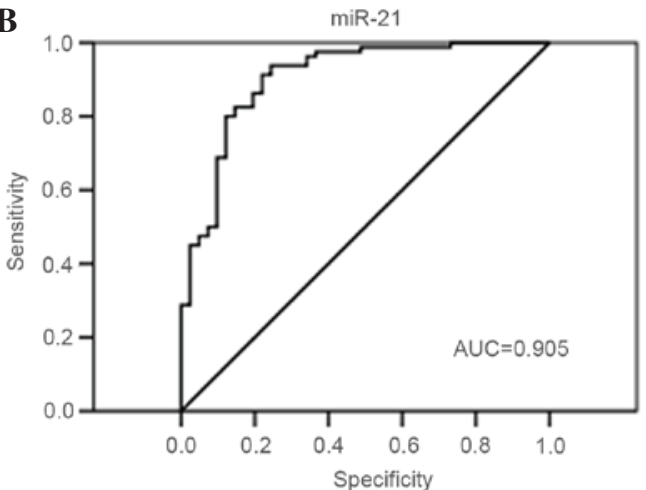

D

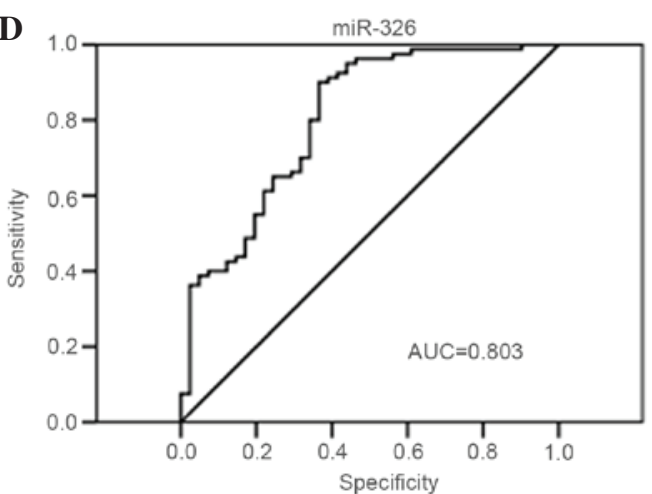

Figure 2. Receiver operating characteristic curves of (A) miR-25, (B) miR-21, (C) miR-27b and (D) miR-326, of the non-benefit and benefit groups. miR-25 showed the highest degree of accuracy (AUC, 0.926). miR, microRNA; AUC, area under the curve.

Prediction of PFS rate using plasma miR expression levels. The association between plasma miR expression levels and PFS rate in 108 patients (benefit group, $n=67$; non-benefit group, $n=41$ ) was investigated. As shown in Fig. 3, the expression levels of miR-25, miR-27b, miR-21 and miR-326 had a significant effect on the PFS rate (all $\mathrm{P} \leq 0.001$, high miR expression vs. low miR expression). Plasma miR expression levels were inversely correlated with PFS, indicating that increased expression of these miRs is associated with decreased PFS.

\section{Discussion}

It is well established that miRs are stable in the blood, and that circulating miRs may act as biomarkers for early diagnosis and prognosis of human cancer (24-32). Likewise, clinical studies 

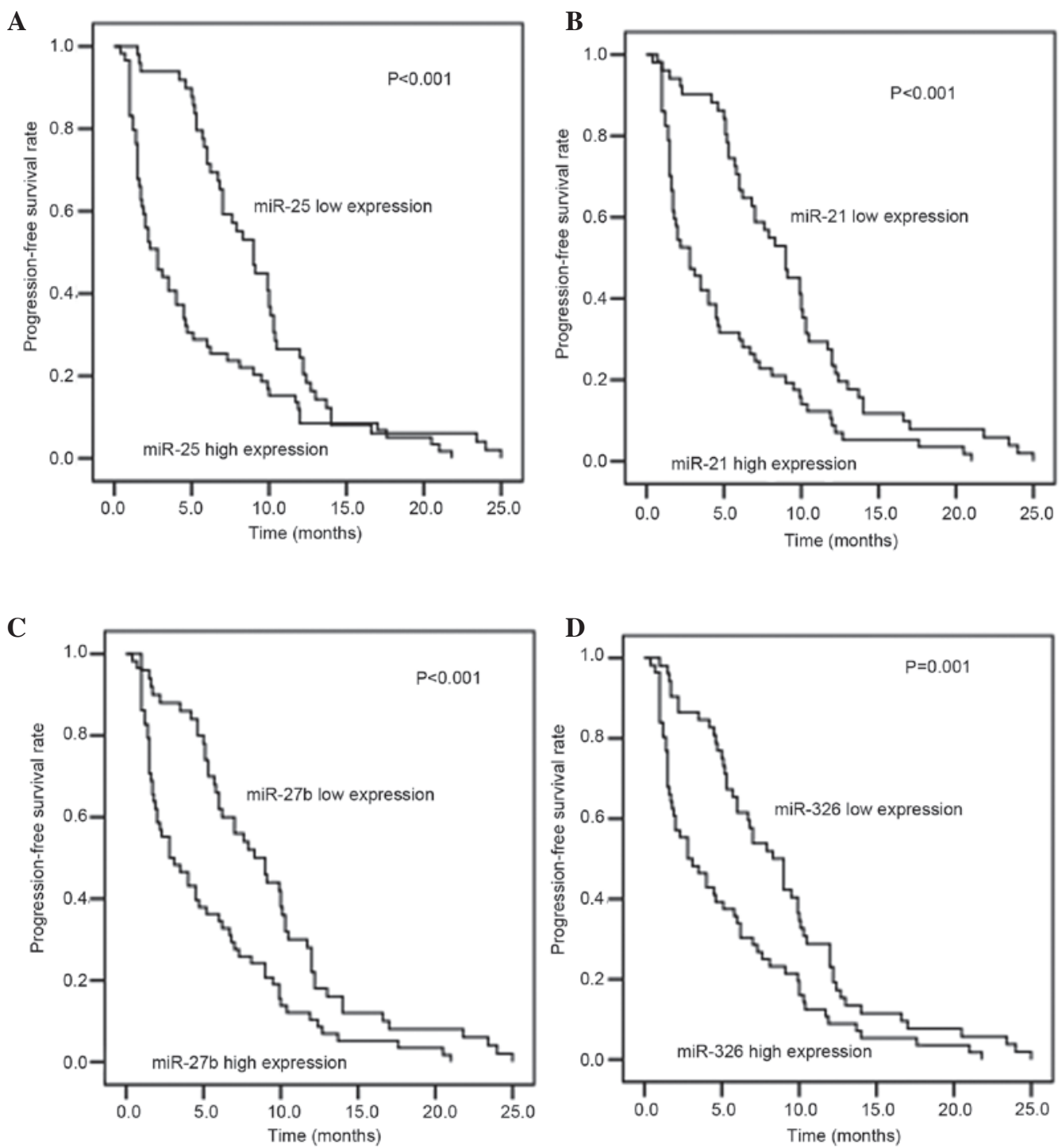

Figure 3. Kaplan-Meier estimation of the progression-free survival of patients with advanced lung adenocarcinoma according to the plasma expression of specific miRs in 108 patients. (A) miR-25, (B) miR-21, (C) miR-27b and (D) miR-326. Increased miR expression levels were associated with decreased progression-free survival. miR, microRNA.

have demonstrated that circulating miRs may serve as predictors of resistance to anticancer agents $(33,34)$. In the present study, plasma miR profiles were compared between benefit and non-benefit groups in order to identify candidate circulating biomarkers that may be used to predict non-benefit from first-line pemetrexed and platinum therapy in patients with advanced LAC.

The expression profile of circulating miRs differs markedly between individuals, disease states, types of cancer and tissues. In the present study, to prevent the influence of this diversity, patient plasma was compared prior to and following chemotherapy using a microarray to measure target miR expression levels. To avoid the compounding effect of nicotine, which may alter the miR expression (42), and comorbidities, including diabetes and hypertension, patients exhibiting these characteristics were excluded from the screening and the training sets. The results in the training set were repeatable in the validation set.

From the results of the miR microarray screening, 6 miRs were selected for further analysis using RT-qPCR. It was observed that in the training and validation sets, the plasma expression levels of miR-25, miR-21, miR-27b and miR-326 prior to treatment were significantly upregulated in the non-benefit group compared with the benefit group. Furthermore, increased expression of these four miRs was associated with poor PFS. The predictive power of each miR was evaluated using ROC curves, in which miR-25 exhibited the highest degree of accuracy (AUC, 0.926; 95\% CI, 0.881-0.971). Among these four miRs, miR-21 is recognized as a marker of reduced therapeutic response and decreased survival in patients with lung cancer (43-45). In addition, miR-21 is associated with multidrug resistance (46), particularly to platinum in NSCLC (47). In the present study, plasma miR-21 expression levels were upregulated in the non-benefit group compared with the benefit group, and high expression of miR-21 was associated with poor PFS.

miR-25 is known to be dysregulated in various types of cancer, and to have oncogenic and tumor suppressive functions $(48,49)$. In NSCLC cell lines and human tissue, miR-25 
expression is significantly increased compared with normal lung cells or adjacent non-cancerous tissues, respectively (50). Downregulation of miR-25 may increase cisplatin sensitivity and suppress the growth of NSCLC cells in vivo (51). Furthermore, increased miR-25 expression has been associated with poor OS in non-smoking females with LAC (52). The results of the present study demonstrate that miR-25 is significantly upregulated in the blood plasma of patients with LAC in the non-benefit group compared with the benefit group, and that high plasma miR-25 expression is associated with decreased PFS.

The expression level of miR-27b varies between different types of cancer. miR-27b may be upregulated or downregulated in chemoresistant cancer cells and tumor samples (53-55). High expression levels of miR-27b in a number of cancer samples have been reported to be associated with good or bad prognoses $(56,57)$. In previous studies miR-27b was found to be downregulated in several NSCLC cell lines and lung cancer tissues $(58,59)$. In the present study, plasma miR-27b was demonstrated to be significantly upregulated in patients with LAC in the non-benefit group compared with the benefit group, and high plasma expression of miR-27b was associated with decreased PFS. Shen et al (60) reported that a number of miRs did not exhibit similar expression patterns in plasma and tumor tissue samples, suggesting that miR expression may be altered by host-derived factors in response to the tumor and metastases, in addition to by the tumor directly.

miR-326 is a suppressor of the Hedgehog, Notch and mitogen-activated protein kinase signaling pathways that are associated with brain tumors (61-63), and may block expression of multidrug resistance-associated proteins in breast cancer (64). Low expression levels of miR-326 are correlated with poor OS in patients with pathological grade III-IV glioma (65). In addition, miR-326 expression was shown to be downregulated in metastatic compared with non-metastatic primary loci in nude mouse NSCLC cells (66). Therefore, it may be possible to use miR-326 expression levels to monitor bone metastasis in patients with LAC (67). The results of the present study are consistent with a previous study, which demonstrated that high plasma expression of miR-27b, miR-148a and miR-326 prior to treatment with 5-fluorouracil and oxaliplatin was correlated with decreased PFS in patients with metastatic colorectal cancer (68).

In the current study, the EGFR genotype of the majority of patients was unknown. Previous studies have identified an interaction between EGFR and miRs (69). For instance, miR-21 is positively regulated by EGFR in cancer cells (70) and the gene encoding EGFR is a potential target of miR-23b/27b (71). Therefore, EGFR genotype status may influence miR expression levels. In the present study, the EGFR genotype status (known or unknown) was similar in the non-benefit and benefit groups, which may have reduced this effect to a certain extent.

In conclusion, the results of the present study demonstrate that overexpression of plasma miR-25, miR-21, miR-27b and miR-326 in patients with advanced LAC is predictive of non-benefit to pemetrexed and platinum therapy, and that increased expression of these miRs is associated with decreased PFS. Among these miRs, miR-25 exhibited the highest degree of accuracy in predicting non-benefit, indicating that it is the most promising biomarker. The results of the current study suggest that plasma miRs may be used as minimally invasive independent molecular biomarkers to predict non-benefit from chemotherapy and PFS rates in patients with advanced LAC.

\section{Acknowledgements}

The authors thank Professor Tingting Wang of the Immunology and Reproduction Biology Laboratory of Nanjing University for her advice.

\section{References}

1. Torre LA, Bray F, Siegel RL, Ferlay J, Lortet-Tieulent J and Jemal A: Global cancer statistics, 2012. CA Cancer J Clin 65: 87-108, 2015

2. Parkin DM, Bray F, Ferlay J and Pisani P: Global cancer statistics, 2002. CA Cancer J Clin 55: 74-108, 2005.

3. Scagliotti GV, Parikh P, von Pawel J, Biesma B, Vansteenkiste J, Manegold C, Serwatowski P, Gatzemeier U, Digumarti R, Zukin M, et al: Phase III study comparing cisplatin plus gemcitabine with cisplatin plus pemetrexed in chemotherapy-naive patients with advanced-stage non-small-cell lung cancer. J Clin Oncol 26: 3543-3551, 2008.

4. Lee JK, Hahn S, Kim DW, Suh KJ, Keam B, Kim TM, Lee SH and Heo DS: Epidermal growth factor receptor tyrosine kinase inhibitors vs conventional chemotherapy in non-small cell lung cancer harboring wild-type epidermal growth factor receptor: A meta-analysis. JAMA 311: 1430-1437, 2014.

5. Scagliotti G, Brodowicz T, Shepherd FA,Zielinski C, Vansteenkiste J, Manegold C, Simms L, Fossella F, Sugarman K and Belani CP: Treatment-by-histology interaction analyses in three phase III trials show superiority of pemetrexed in nonsquamous non-small cell lung cancer. J Thorac Oncol 6: 64-70, 2011.

6. Pennell NA: Selection of chemotherapy for patients with advanced non-small cell lung cancer. Cleve Clin J Med 79 Electronic (Suppl 1): eS46-eS50, 2012.

7. Kim YH, Hirabayashi M, Togashi Y, Hirano K, Tomii K, Masago K, Kaneda T, Yoshimatsu H, Otsuka K, Mio T, et al: Phase II study of carboplatin and pemetrexed in advanced non-squamous, non-small-cell lung cancer: Kyoto thoracic oncology research group trial 0902. Cancer Chemother Pharmacol 70: 271-276, 2012.

8. Hartmann JT and Lipp HP: Toxicity of platinum compounds. Expert Opin Pharmacother 4: 889-901, 2003.

9. Gota V, Kavathiya K, Doshi K, Gurjar M, Damodaran SE, Noronha V, Joshi A and Prabhash K: High plasma exposure to pemetrexed leads to severe hyponatremia in patients with advanced non small cell lung cancer receiving pemetrexed-platinum doublet chemotherapy. Cancer Manag Res 6: 261-265, 2014.

10. Olaussen KA, Dunant A, Fouret P, Brambilla E, André F, Haddad V, Taranchon E, Filipits M, Pirker R, Popper HH, et al: DNA repair by ERCC1 in non-small-cell lung cancer and cisplatin-based adjuvant chemotherapy. N Engl J Med 355: 983-991, 2006.

11. Cobo M, Isla D, Massuti B, Montes A, Sanchez JM, Provencio M, Viñolas N, Paz-Ares L, Lopez-Vivanco G, Muñoz MA, et al: Customizing cisplatin based on quantitative excision repair cross-complementing $1 \mathrm{mRNA}$ expression: A phase III trial in non-small-cell lung cancer. J Clin Oncol 25: 2747-2754, 2007.

12. Boukovinas I, Papadaki C, Mendez P, Taron M, Mavroudis D, Koutsopoulos A, Sanchez-Ronco M, Sanchez JJ, Trypaki M, Staphopoulos E, et al: Tumor BRCA1, RRM1 and RRM2 mRNA expression levels and clinical response to first-line gemcitabine plus docetaxel in non-small-cell lung cancer patients. PLoS One 3: e3695, 2008.

13. Carser JE, Quinn JE, Michie CO, O'Brien EJ, McCluggage WG, Maxwell P, Lamers E, Lioe TF, Williams AR, Kennedy RD, et al: BRCA1 is both a prognostic and predictive biomarker of response to chemotherapy in sporadic epithelial ovarian cancer. Gynecol Oncol 123: 492-498, 2011.

14. Nicolson MC, Fennell DA, Ferry D, O'Byrne K, Shah R, Potter V, Skailes G, Upadhyay S, Taylor P, André V, et al: Thymidylate synthase expression and outcome of patients receiving pemetrexed for advanced nonsquamous non-small-cell lung cancer in a prospective blinded assessment phase II clinical trial. J Thorac Oncol 8: 930-939, 2013 
15. Friboulet L, Olaussen KA, Pignon JP, Shepherd FA, Tsao MS, Graziano S, Kratzke R, Douillard JY, Seymour L, Pirker R, et al: ERCC1 isoform expression and DNA repair in non-small-cell lung cancer. N Engl J Med 368: 1101-1110, 2013.

16. Bepler G, Williams C, Schell MJ, Chen W, Zheng Z, Simon G, Gadgeel S, Zhao X, Schreiber F, Brahmer J, et al: Randomized international phase III trial of ERCC1 and RRM1 expression-based chemotherapy versus gemcitabine/carboplatin in advanced non-small-cell lung cancer. J Clin Oncol 31: 2404-2412, 2013.

17. Moran T, Wei J, Cobo M, Qian X, Domine M, Zou Z, Bover I, Wang L, Provencio M, Yu L, et al: Two biomarker-directed randomized trials in European and Chinese patients with nonsmall-cell lung cancer: The BRCA1-RAP80 Expression Customization (BREC) studies. Ann Oncol 25: 2147-2155, 2014.

18. Kerr KM, Bubendorf L, Edelman MJ, Marchetti A, Mok T, Novello S, O'Byrne K, Stahel R, Peters S and Felip E; Panel Members; Panel Members: Second ESMO consensus conference on lung cancer: Pathology and molecular biomarkers for non-small-cell lung cancer. Ann Oncol 25: 1681-1690, 2014

19. Lewis BP, Burge CB and Bartel DP: Conserved seed pairing, often flanked by adenosines, indicates that thousands of human genes are microRNA targets. Cell 120: 15-20, 2005.

20. Farazi TA, Spitzer JI, Morozov P and Tuschl T: miRNAs in human cancer. J Pathol 223: 102-115, 2011.

21. Wang J, Tian X, Han R, Zhang X, Wang X, Shen H, Xue L, Liu Y, Yan X, Shen J, et al: Downregulation of miR-486-5p contributes to tumor progression and metastasis by targeting protumorigenic ARHGAP5 in lung cancer. Oncogene 33: $1181-1189,2014$

22. Fuse M, Kojima S, Enokida H, Chiyomaru T, Yoshino H, Nohata N, Kinoshita T, Sakamoto S, Naya Y, Nakagawa M, et al: Tumor suppressive microRNAs (miR-222 and miR-31) regulate molecular pathways based on microRNA expression signature in prostate cancer. J Hum Genet 57: 691-699, 2012.

23. Selcuklu SD, Donoghue MT and Spillane C: miR-21 as a key regulator of oncogenic processes. Biochem Soc Trans 37: 918-925, 2009

24. Chen X, Ba Y, Ma L, Cai X, Yin Y, Wang K, Guo J, Zhang Y, Chen J, Guo X, et al: Characterization of microRNAs in serum: A novel class of biomarkers for diagnosis of cancer and other diseases. Cell Res 18: 997-1006, 2008.

25. Mitchell PS, Parkin RK, Kroh EM, Fritz BR, Wyman SK, Pogosova-Agadjanyan EL, Peterson A, Noteboom J, O'Briant KC, Allen A, et al: Circulating microRNAs as stable blood-based markers for cancer detection. Proc Natl Acad Sci USA 105: 10513-10518, 2008.

26. Rabinowits G, Gerçel-Taylor C, Day JM, Taylor DD and Kloecker GH: Exosomal microRNA: A diagnostic marker for lung cancer. Clin Lung Cancer 10: 42-46, 2009.

27. Huang Z, Huang D, Ni S, Peng Z, Sheng W and Du X: Plasma microRNAs are promising novel biomarkers for early detection of colorectal cancer. Int J Cancer 127: 118-126, 2010.

28. Toiyama Y, Hur K, Tanaka K, Inoue Y, Kusunoki M, Boland CR and Goel A: Serum miR-200c is a novel prognostic and metastasis-predictive biomarker in patients with colorectal cancer. Ann Surg 259: 735-743, 2014.

29. Eichelser C, Flesch-Janys D, Chang-Claude J, Pantel K and Schwarzenbach H: Deregulated serum concentrations of circulating cell-free microR NAs miR-17, miR-34a, miR-155, and miR-373 in human breast cancer development and progression. Clin Chem 59: 1489-1496, 2013.

30. Zhu C, Ren C, Han J, Ding Y, Du J, Dai N, Dai J, Ma H, Hu Z and Shen $\mathrm{H}$, et al: A five-microRNA panel in plasma was identified as potential biomarker for early detection of gastric cancer. Br J Cancer 110: 2291-2299, 2014.

31. Yang C, Wang C, Chen X, Chen S, Zhang Y, Zhi F, Wang J, Li L, Zhou X, Li N, et al: Identification of seven serum microRNAs from a genome-wide serum microRNA expression profile as potential noninvasive biomarkers for malignant astrocytomas. Int J Cancer 132: 116-127, 2013.

32. Zheng H, Zhang L, Zhao Y, Yang D, Song F, Wen Y, Hao Q, $\mathrm{Hu} \mathrm{Z}$, Zhang $\mathrm{W}$ and Chen K: Plasma miRNAs as diagnostic and prognostic biomarkers for ovarian cancer. PLoS One 8: e77853, 2013.

33. Nolen BM, Marks JR, Ta'san S, Rand A, Luong TM, Wang Y, Blackwell K and Lokshin AE: Serum biomarker profiles and response to neoadjuvant chemotherapy for locally advanced breast cancer. Breast Cancer Res 10: R45, 2008.
34. Hansen TF, Sørensen FB, Lindebjerg J and Jakobsen A: The predictive value of microRNA-126 in relation to first line treatment with capecitabine and oxaliplatin in patients with metastatic colorectal cancer. BMC Cancer 12: 83, 2012

35. Seidman AD, O'Shaughnessy J and Misset JL: Single-agent capecitabine: A reference treatment for taxane-pretreated metastatic breast cancer. Oncologist 7: (Suppl 6): 20-28, 2002.

36. Cecchin E, Innocenti F, D'Andrea M, Corona G, De Mattia E, Biason P, Buonadonna A and Toffoli G: Predictive role of the UGT1A1, UGT1A7, and UGT1A9 genetic variants and their haplotypes on the outcome of metastatic colorectal cancer patients treated with fluorouracil, leucovorin, and irinotecan. J Clin Oncol 27: 2457-2465, 2009.

37. Edge S, Byrd DR, Compton CC, Fritz AG, Greene FL and Trotti A (eds): AJCC Cancer Staging Manual. 7th edition. Springer-Verlag, New York, NY, 2010.

38. Eisenhauer EA, Therasse P, Bogaerts J, Schwartz LH, Sargent D, Ford R, Dancey J, Arbuck S, Gwyther S, Mooney M, et al: New response evaluation criteria in solid tumours: Revised RECIST guideline (version 1.1). Eur J Cancer 45: 228-247, 2009.

39. Churchill GA: Fundamentals of experimental design for cDNA microarrays. Nat Genet 32: (Suppl) 490-495, 2002.

40. Livak KJ and Schmittgen TD: Analysis of relative gene expression data using real-time quantitative PCR and the 2(-Delta Delta C(T)) Method. Methods 25: 402-408, 2001.

41. Youden WJ: Index for rating diagnostic tests. Cancer 3: 32-35, 1950.

42. Ng TK, Carballosa CM, Pelaez D, Wong HK, Choy KW, Pang CP and Cheung HS: Nicotine alters MicroRNA expression and hinders human adult stem cell regenerative potential. Stem Cells Dev 22: 781-790, 2013

43. Liu ZL, Wang H, Liu J and Wang ZX: MicroRNA-21 (miR-21) expression promotes growth, metastasis, and chemo- or radioresistance in non-small cell lung cancer cells by targeting PTEN. Mol Cell Biochem 372: 35-45, 2013.

44. Gao W, Lu X, Liu L, Xu J, Feng D and Shu Y: MiRNA-21: A biomarker predictive for platinum-based adjuvant chemotherapy response in patients with non-small cell lung cancer. Cancer Biol Ther 13: 330-340, 2012.

45. Liu XG, Zhu WY, Huang YY, Ma LN, Zhou SQ, Wang YK, Zeng F, Zhou JH and Zhang YK: High expression of serum miR-21 and tumor miR-200c associated with poor prognosis in patients with lung cancer. Med Oncol 29: 618-626, 2012.

46. Dong Z, Ren L, Lin L, Li J, Huang Y and Li J: Effect of microRNA-21 on multidrug resistance reversal in A549/DDP human lung cancer cells. Mol Med Rep 11: 682-690, 2015.

47. Xu L, Huang Y, Chen D, He J, Zhu W, Zhang Y and Liu X: Downregulation of miR-21 increases cisplatin sensitivity of non-small-cell lung cancer. Cancer Genet 207: 214-220, 2014

48. Zhang H, Zuo Z, Lu X, Wang L, Wang H and Zhu Z: MiR-25 regulates apoptosis by targeting Bim in human ovarian cancer. Oncol Rep 27: 594-598, 2012.

49. Li Q, Zou C, Zou C, Han Z, Xiao H, Wei H, Wang W, Zhang L, Zhang X, Tang Q, et al: MicroRNA-25 functions as a potential tumor suppressor in colon cancer by targeting Smad7. Cancer Lett 335: 168-174, 2013.

50. Yang T, Chen T, Li Y, Gao L, Zhang S, Wang T and Chen M: Downregulation of miR-25 modulates non-small cell lung cancer cells by targeting CDC42. Tumour Biol 36: 1903-1911, 2015.

51. Yang T, Chen T, Li Y, Gao L, Zhang S, Wang T and Chen M: Downregulation of miR-25 modulates non-small cell lung cancer cells by targeting CDC42. Tumour Biol 36: 1903-1911, 2015.

52. Xu FX, Su YL, Zhang H, Kong JY, Yu H and Qian BY: Prognostic implications for high expression of MiR-25 in lung adenocarcinomas of female non-smokers. Asian Pac J Cancer Prev 15: 1197-1203, 2014.

53. Rasmussen MH, Jensen NF, Tarpgaard LS, Qvortrup C, Rømer MU, Stenvang J, Hansen TP, Christensen LL, Lindebjerg J, Hansen F, et al: High expression of microRNA-625-3p is associated with poor response to first-line oxaliplatin based treatment of metastatic colorectal cancer. Mol Oncol 7: 637-646, 2013.

54. Park YT, Jeong JY, Lee MJ, Kim KI, Kim TH, Kwon YD, Lee C, Kim OJ and An HJ: MicroRNAs overexpressed in ovarian ALDH1-positive cells are associated with chemoresistance. J Ovarian Res 6: 18, 2013.

55. Bera A, VenkataSubbaRao K, Manoharan MS, Hill P and Freeman JW: A miRNA signature of chemoresistant mesenchymal phenotype identifies novel molecular targets associated with advanced pancreatic cancer. PLoS One 9: e106343, 2014. 
56. Buffa FM, Camps C, Winchester L, Snell CE, Gee HE, Sheldon H Taylor M, Harris AL and Ragoussis J: microRNA-associated progression pathways and potential therapeutic targets identified by integrated mRNA and microRNA expression profiling in breast cancer. Cancer Res 71: 5635-5645, 2011.

57. Goto Y, Kojima S, Nishikawa R, Enokida H, Chiyomaru T, Kinoshita T, Nakagawa M, Naya Y, Ichikawa T and Seki N: The microRNA-23b/27b/24-1 cluster is a disease progression marker and tumor suppressor in prostate cancer. Oncotarget 5 : $7748-7759,2014$

58. Jiang J, Lv X, Fan L, Huang G, Zhan Y, Wang M and Lu H: MicroRNA-27b suppresses growth and invasion of NSCLC cells by targeting Sp1. Tumour Biol 35: 10019-10023, 2014.

59. Yanaihara N, Caplen N, Bowman E, Seike M, Kumamoto K Yi M, Stephens RM, Okamoto A, Yokota J, Tanaka T, et al: Unique microRNA molecular profiles in lung cancer diagnosis and prognosis. Cancer Cell 9: 189-198, 2006.

60. Shen J, Todd NW, Zhang H, Yu L, Lingxiao X, Mei Y, Guarnera M, Liao J, Chou A, Lu CL, et al: Plasma microRNAs as potential biomarkers for non-small-cell lung cancer. Lab Invest 91: 579-587, 2011.

61. Kefas B, Comeau L, Floyd DH, Seleverstov O, Godlewski J, Schmittgen T, Jiang J, diPierro CG, Li Y, Chiocca EA, et al: The neuronal microRNA miR-326 acts in a feedback loop with notch and has therapeutic potential against brain tumors. J Neurosci 29: 15161-15168, 2009.

62. Ferretti E, De Smaele E, Miele E, Laneve P, Po A, Pelloni M, Paganelli A, Di Marcotullio L, Caffarelli E, Screpanti I, et al: Concerted microRNA control of Hedgehog signalling in cerebellar neuronal progenitor and tumour cells. EMBO J 27: 2616-2627, 2008

63. Zhou J, Xu T, Yan Y, Qin R, Wang H, Zhang X, Huang Y, Wang Y, Lu Y, Fu D and Chen J: MicroRNA-326 functions as a tumor suppressor in glioma by targeting the Nin one binding protein (NOB1). PLoS One 8: e68469, 2013.
64. Liang Z, Wu H, Xia J, Li Y, Zhang Y, Huang K, Wagar N, Yoon Y, Cho HT, Scala S and Shim H: Involvement of miR-326 in chemotherapy resistance of breast cancer through modulating expression of multidrug resistance-associated protein 1. Biochem Pharmacol 79: 817-824, 2010.

65. Wang S, Lu S, Geng S, Ma S, Liang Z and Jiao B: Expression and clinical significance of microRNA-326 in human glioma miR-326 expression in glioma. Med Oncol 30: 373, 2013.

66. Wang R, Chen XF and Shu YQ: Prediction of non-small cell lung cancer metastasis-associated microRNAs using bioinformatics. Am J Cancer Res 5: 32-51, 2014. eCollection 2015.

67. Valencia K, Martín-Fernández M, Zandueta C, Ormazábal C, Martínez-Canarias S, Bandrés E, de la Piedra C and Lecanda F: miR-326 associates with biochemical markers of bone turnover in lung cancer bone metastasis. Bone 52: 532-539, 2013.

68. Kjersem JB, Ikdahl T, Lingjaerde OC, Guren T, Tveit KM and Kure EH: Plasma microRNAs predicting clinical outcome in metastatic colorectal cancer patients receiving first-line oxaliplatin-based treatment. Mol Oncol 8: 59-67, 2014.

69. Gomez GG, Wykosky J, Zanca C, Furnari FB and Cavenee WK: Therapeutic resistance in cancer: microRNA regulation of EGFR signaling networks. Cancer Biol Med 10: 192-205, 2013.

70. Seike M, Goto A, Okano T, Bowman ED, Schetter AJ, Horikawa I, Mathe EA, Jen J, Yang P, Sugimura H, Gemma A, Kudoh S, Croce CM and Harris CC: MiR-21 is an EGFR-regulated anti-apoptotic factor in lung cancer in never-smokers. Proc Natl Acad Sci USA 106: 12085-12090, 2009.

71. Chiyomaru T, Seki N, Inoguchi S, Ishihara T, Mataki H, Matsushita R, Goto Y, Nishikawa R, Tatarano S, Itesako T, et al: Dual regulation of receptor tyrosine kinase genes EGFR and c-Met by the tumor-suppressive microRNA-23b/27b cluster in bladder cancer. Int J Oncol 46: 487-496, 2015. 